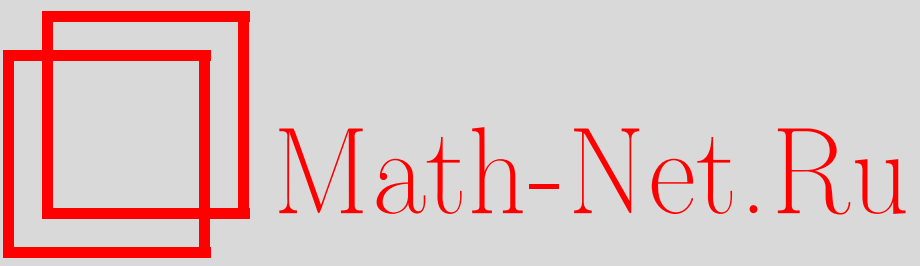

А. И. Зенчук, Преобразования типа Миуры нелинейных уравнений в частных производных, интегрируемых методом $\bar{\partial}$-проблемы, ТМФ, 1999, том 119, номер 1, 47-54

DOI: https://doi.org/10.4213/tmf726

Использование Общероссийского математического портала Math-Net.Ru подразумевает, что вы прочитали и согласны с пользовательским соглашением

http://www.mathnet.ru/rus/agreement

Параметры загрузки:

IP : 54.196 .121 .252

26 апреля 2023 г., 14:51:42 


\section{ПРЕОБРАЗОВАНИЯ ТИПА МИУРЫ НЕЛИНЕЙНЫХ УРАВНЕНИЙ В ЧАСТНЫХ ПРОИЗВОДНЫХ, ИНТЕГРИРУЕМЫХ МЕТОДОМ $\overline{-}$ ПРОБЛЕМЫ}

Предложен новый способ построения преобразований типа Миуры для систем нелинейных уравнений математической физики, интегрируемых методом $\bar{\partial}$-проблемы, с помощью введения обобщенной нормировочной функции в $\bar{\partial}$-проблему. Рассмотрены примеры таких преобразований для уравнения Кадомцева-Петвиашвили.

\section{1. ВВЕДЕНИЕ}

В теории интегрируемости нелинейных уравнений математической физики фундаментальная роль отводится методу одевания [1] как регулярному методу построения решений интегрируемых нелинейных дифференциальных уравнений в частных производных, имеющих приложение в различных областях физики. Современным вариантом метода одевания является нелокальная $\bar{\partial}$-проблема $[2,3]$, которая позволяет строить определенные классы решений $(2+1)$-мерных нелинейных уравнений.

Под нелокальной $\bar{\partial}$-проблемой $[2,3]$ понимается интегро-дифференшиальное уравнение

$$
\begin{aligned}
\bar{\partial} \psi_{p}(\lambda ; \mathbf{x}) & =Q_{p}(\lambda ; \mathbf{x})+\int \psi_{p}(\mu ; \mathbf{x}) R(\mu, \lambda ; \mathbf{x}) d^{2} \mu \\
Q_{p} & =\bar{\partial} q_{p}, \quad \mathbf{x}=\left(x_{1}, \ldots, x_{N}\right) \\
\left.\psi_{p}(\lambda ; \mathbf{x})\right|_{\lambda \rightarrow \infty} & \rightarrow q_{p}(\lambda ; \mathbf{x})+\sum_{k} \frac{\psi_{p k}(\mathbf{x})}{\lambda^{k}}, \quad p=1, \ldots, N_{1}, \quad N_{1} \in \mathbf{Z},
\end{aligned}
$$

где все функции матричнозначные, величины $q_{p}$ называются нормировочными функциями (или нормировками), ядро $R$ - произвольная функция своих аргументов, обеспечивающая однозначную разрешимость уравнения (1); $\psi_{p}$ - решения уравнения (1) с одним и тем же ядром $R$, но с разными нормировочными функциями $q_{p}$. В дальнейшем аргументы в скобках по возможности будем опускать.

${ }^{*}$ Институт теоретической физики им. Л. Д. Ландау РАН, Москва, Россия. E-mail: zenchuk@itp.ac.ru 
Напомним, что ранее [2-4] для построения интегрируемых уравнений использовались решения уравнения (1) с дробно-рациональными нормировочными функциями вида

$$
q_{p}=P_{p}(\lambda)+\sum_{k} \frac{f_{p k}(\mathbf{x})}{\lambda-a_{p k}}
$$

где $P_{p}(\lambda)$ - полиномы по $\lambda$, коэффициенты которых произвольным образом зависят от $\mathbf{x}$, $a_{p k}$ - некоторые точки комплексной плоскости, $f_{p k}-$ произвольные функции $\mathbf{x}$. Зависимость от параметров х вводилась с помощью уравнений

$$
R_{x_{i}}(\lambda, \mu)=K_{x_{i}}(\lambda ; \mathbf{x}) R(\lambda, \mu)-R(\lambda, \mu) K_{x_{i}}(\mu ; \mathbf{x})
$$

где одеваюший оператор $K$ - некоторая матричнозначная дробно-рациональная функция $\lambda$, линейная по х.

В данной работе мы расширим класс допустимых нормировочных функций и рассмотрим функции $q_{p}$ с произвольной зависимостью от параметра $\lambda$ и фиксированной зависимостью от параметров $\mathbf{x}$, задаваемой некоторой системой дифференциальных уравнений. Эта зависимость вместе с уравнением (3) определяет конкретный вид нелинейной системы уравнений, описываемой задачей (1). Такая модификация $\bar{\partial}$-проблемы сушественно расширяет класс уравнений, интегрируемых с ее помощью.

Важен тот факт, что между системами нелинейных уравнений, описываемых $\bar{\partial}$-проблемой с одним и тем же ядром $R$, но с разными нормировочными функциями, существует связь типа преобразований Миуры, о чем будет говориться в конце раздела 2. Таким образом, уравнение (1) с обобщенной нормировочной функцией реализует новьй способ построения преобразований типа Миуры известных интегрируемых систем. В разделе 3 разобрано несколько примеров таких преобразований для уравнения Кадомцева-Петвиашвили (КП).

Отметим, что приводимые рассуждения справедливы и для локальной $\bar{\partial}$-проблемы.

\section{2. СХЕМА ПОСТРОЕНИЯ НЕЛИНЕЙНЫХ УРАВНЕНИЙ. ПРЕОБРАЗОВАНИЯ ТИПА МИУРЫ}

Как известно $[1,2]$, с решением $\phi$ задачи $(1)$, нормированным на функцию $q_{0}$ вида $(2)$, связано множество решений $\phi^{(n)}=M_{n} \phi$ уравнения (1) с другими нормировками $q_{0 n}$ вида (2), которые строятся из функции $\phi$ с помошью операторов

$$
M_{n}=\sum_{k} v_{k}(\mathbf{x}) \prod_{n} D_{x_{n}}^{m_{n k}}
$$

Операторы $D_{x_{n}}$ действуют на произвольную функцию $\phi$ по формуле $D_{x_{n}} \phi=\phi_{x_{n}}+$ $\phi K_{x_{n}}, v_{k}-$ произвольные функции параметров $\mathbf{x}$. Обозначим множество таких операторов через $\widetilde{M}$ :

$$
\widetilde{M}=\left\{M_{k}: \bar{\partial}\left(M_{k} \phi\right)(\lambda)=\bar{\partial} q_{0 k}+\int\left(M_{k} \phi\right)(\mu) R(\mu, \lambda) d^{2} \mu, k \in \mathbf{Z}\right\} .
$$


Нелинейные уравнения возникают для функций $\phi_{k}, \phi\left(b_{k}\right)$, где $\phi_{k}-$ коэффициенты степенного ряда по $1 / \lambda$,

$$
\left.\phi\right|_{\lambda \rightarrow \infty} \rightarrow q_{0}+\sum_{k} \frac{\phi_{k}}{\lambda^{k}},
$$

$b_{k}$ - полюсы оператора $K$. Конкретный вид уравнений определяется оператором $K$ в уравнениях (3). Их построение основано на нахождении линейных дифференциальных операторов $\widetilde{M}_{k}$ (с коэффициентами, зависяшими от х) из множества $\widetilde{M}$ таких, что функции $\chi_{k}=\widetilde{M}_{k} \phi$ являются решениями уравнения (1) с нормировкой $q_{0 k}=0$. Вследствие однозначной разрешимости уравнения (1) это означает, что

$$
\widetilde{M}_{k} \phi \equiv 0 .
$$

Обозначим множество таких операторов через $\widetilde{M}_{0}$ :

$$
\widetilde{M}_{0}=\left\{\widetilde{M}_{k}: \widetilde{M}_{k} \phi=0, k \in \mathbf{Z}\right\} .
$$

Его сушествование обусловлено уравнениями (3). Согласно результатам работ $[2,3]$ существуют только два независимых оператора из множества $\widetilde{M}_{0}$ на каждую тройку независимых переменных $x_{i}, x_{j}, x_{k}$ из множества $\mathbf{x}$, которые представляют собой вспомогательную переопределенную линейную систему уравнений с переменными коэффициентами на функции $\psi_{p}$.

Подробный алгоритм построения нелинейных дифференциальных уравнений в частных производных, интегрируемых методом $\bar{\partial}$-проблемы (1), (2), приведен в работах $[2,3]$. Прежде чем применить его к уравнению (1) с нормировками $q_{p}\left(p=1, \ldots, N_{1}\right)$, произвольно зависяшими от $\lambda$, необходимо ввести конкретную зависимость величин $q_{p}$ от параметров х. Пусть эта зависимость определяется системой уравнений

$$
\sum_{p=1}^{N_{1}} L_{p j} Q_{p}=\sum_{k=1}^{N_{2}} \alpha_{j k}(\mathbf{x}) \delta\left(\lambda-a_{j k}\right), \quad j=1, \ldots, N_{3}, \quad N_{1}, N_{2}, N_{3} \in \mathbf{Z}
$$

где $L_{p j}$ - линейные дифференшиальные операторы с коэффишиентами, зависяшими от $\lambda$ и $\mathbf{x}, \alpha_{j k}$-некоторые функции параметров $\mathbf{x}, a_{j k}$-некоторые точки комплексной плоскости. Пусть множеству операторов $L_{p j}$ соответствует множество линейных дифференциальных операторов $T_{p j}\left(p=1, \ldots, N_{1}, j=1, \ldots, N_{3}\right)$ таких, что функции

$$
\chi_{j}=\sum_{p=1}^{N_{1}} T_{p j} \psi_{p} \equiv T_{j}(\psi), \quad \psi=\left(\psi_{1}, \ldots, \psi_{N_{1}}\right),
$$

являются решениями уравнения

$$
\bar{\partial} \chi_{j}(\lambda)=\sum_{p=1}^{N_{1}} L_{p j} Q_{p}+\int \chi_{j}(\mu) R(\mu, \lambda) d^{2} \mu .
$$


С учетом системы (7) это означает, что функция $\chi_{j}$ есть решение уравнения (1) с нормировкой $q_{j}^{(\chi)}$ :

$$
q_{j}^{(\chi)}=P_{j}(\lambda)+\sum_{k=1}^{N_{2}} \frac{\alpha_{j k}(\mathbf{x})}{\lambda-a_{j k}},\left.\quad \chi_{j}\right|_{\lambda \rightarrow \infty} \rightarrow q_{j}^{(\chi)}+\sum_{k \geqslant 0} \frac{\chi_{j k}}{\lambda^{k}},
$$

где $P_{j}(\lambda)$ - полиномы по $\lambda$, коэффициенты которых (функции $\mathbf{x}$ ) выражаются через $\psi_{p k}$ (см. (1)). В этом случае множество $\widetilde{M}(4)$ состоит из операторов, действуюших на векторную функцию $\psi$ (см. (8)),

$$
\widetilde{M}=\left\{T_{j}, \sum_{k} g_{k}(\mathbf{x}) \prod_{n} D_{x_{n}}^{m_{n k}} T_{j}, m_{n k} \in \mathbf{Z}, j=1, \ldots, N_{3}\right\},
$$

где $g_{k}-$ произвольные функции параметров $\mathbf{x}$.

Разумеется, множество операторов $T_{p j}$ можно подобрать не для любого множества $L_{p j}$. По сути, зависимость коэффициентов допустимых операторов $L_{p j}$ от $\lambda$ определяется одевающим оператором $K$ в уравнении (3) и не может быть произвольной. На зависимость от параметров $\mathbf{x}$ ограничений не накладывается.

Как было сказано вьше, множество $\widetilde{M}(11)$ порождает множество $\widetilde{M}_{0}(6)$, т.е. линейную переопределенную систему уравнений на функции $\psi_{p}$ и произвольную тройку независимых переменных $x_{i}, x_{j}, x_{k}$. При этом нелинейная система уравнений возникает для каждой тройки параметров $x_{i}, x_{j}, x_{k}$ и функций $\psi_{p k}, \psi_{p}\left(b_{k}\right), k, p \in \mathbf{Z}$, где $b_{k}$ полюсы оператора $K$. Чтобы получить эту систему, достаточно разложить линейную систему уравнений, которой удовлетворяют функции $\psi_{p}$, по малым параметрам

$$
\varepsilon_{0}=\left.\frac{1}{\lambda}\right|_{\lambda \rightarrow \infty}, \quad \varepsilon_{k}=\left.\left(\lambda-b_{k}\right)\right|_{\lambda \rightarrow b_{k}}, \quad k=1, \ldots, N_{2},
$$

и приравнять нулю достаточное количество членов этого разложения, для того чтобы получить замкнутую систему уравнений.

Преобразования типа Миуры возникают между системами нелинейных уравнений, порождаемыми функциями $\psi_{p}\left(p=1, \ldots, N_{1}\right)$, с одной стороны, и функциями $\chi_{j}(j=$ $\left.1, \ldots, N_{3}\right)$, с другой. Действительно, поскольку функции $\chi_{j}$ имеют нормировку вида $(2)$, они могут быть использованы для построения нелинейных систем уравнений на функции $\chi_{j k}, \chi_{j}\left(b_{k}\right)$ с учетом уравнений $(3)$ по обычному алгоритму $[2,3]$. В то же время согласно формулам (8) сушествует явная связь между функциями $\psi_{p}$ и $\chi_{j}$. Поэтому, записав уравнения (8) в виде разложений по степеням параметров $\varepsilon_{k}\left(k=0, \ldots, N_{2}\right)(12)$ и приравняв нулю достаточное количество членов этих разложений, получим в результате замкнутую систему дифференциальных уравнений, связывающую между собой функции $\chi_{j k}, \chi\left(b_{k}\right)$ и $\psi_{p k}, \psi\left(b_{k}\right)$. Это и есть искомое преобразование типа Миуры. Очевидно, что подобные преобразования сушествуют и между решениями нелинейных уравнений, порождаемыми $\bar{\partial}$-проблемой с различными нормировочными функциями $q_{p}$ ( $p=$ $\left.1, \ldots, N_{1}\right)$ и $\tilde{q}_{j}\left(j=1, \ldots, N_{3}\right)$, также и в том случае, если ни одна из них не является дробно-рациональной функцией вида (2).

В следующем разделе с помощью введенного алгоритма будут получены некоторые преобразования типа Миуры для уравнения КП. 


\section{3. ПРЕОБРАЗОВАНИЯ МИУРЫ УРАВНЕНИЯ КП}

Во всех примерах этого раздела рассматривается скалярная задача (1). Зависимость ядра от дополнительных параметров $x_{1}=x, x_{2}=y, x_{3}=t$ введем согласно (3) с помощью одеваюшего оператора $K=\lambda x+\lambda^{2} y+\lambda^{3} t$,

$$
R_{x}=\left(\lambda^{\prime}-\lambda\right) R, \quad R_{y}=\left(\lambda^{\prime 2}-\lambda^{2}\right) R, \quad R_{t}=\left(\lambda^{\prime 3}-\lambda^{3}\right) R .
$$

В дальнейшем используются операторы

$$
D_{x}=\partial_{x}+\lambda, \quad D_{y}=\partial_{y}+\lambda^{2}, \quad D_{t}=\partial_{t}+\lambda^{3} .
$$

Приводимые ниже примеры отличаются друг от друга зависимостью нормировок $q_{p}$ от параметров х. Везде в качестве операторов $L_{p j}$ выбраны линейные дифференциальные операторы с коэффициентами, не зависяшими от $\mathbf{x}$.

В примерах 1-3 (приведенных ниже) $N_{1}=1$ (т.е. система (1) состоит из одного уравнения) и приняты обозначения

$$
Q_{1}=Q, \quad \psi_{1}=\psi,\left.\quad \psi\right|_{\lambda \rightarrow \infty} \rightarrow q+\sum_{k>0} \frac{\psi_{k}}{\lambda_{k}}
$$

Первый пример соответствует преобразованию Миуры, связывающему между собой решения уравнения КП и модифицированного уравнения КП. В двух других примерах получаются нелинейные системы, каждая из которых состоит из уравнения КП и линейного уравнения с переменными коэффициентами вида $M\left(L_{-}, A_{-}\right) \phi=0$, причем оператор $M$ есть суперпозищия (вообше говоря, нелинейная, как показано в примере 3 ) операторов $L_{-}$и $A_{-}$,

$$
L_{ \pm}= \pm \partial_{y}-\partial_{x}^{2}+2 u, \quad A_{ \pm}=\partial_{t}-\partial_{x}^{3}+3 u \partial_{x}+\frac{3}{2}\left(u_{x} \pm \partial_{x}^{-1} u_{y}\right),
$$

условие коммутативности которых порождает уравнение КП.

Более конструктивны примеры 4 и 5 , в которых $N_{1}=2$ (два уравнения в системе (1)). При этом получаются нелинейные системы, связанные некоторым преобразованием с уравнением КП.

ПримеР 1. Преобразование Миуры

$$
u=\frac{1}{2}\left(\partial_{x}^{-1} r_{y}+r_{x}+r^{2}\right)
$$

переводящее решения модифищированного уравнения КП

$$
r_{t}-\frac{1}{4} r_{x x x}-\frac{3}{4} \partial_{x}^{-1} r_{y y}+\frac{3}{2} r_{x} \partial_{x}^{-1} r_{y}+\frac{3}{2} r^{2} r_{x}=0
$$

в решения уравнения КП

$$
u_{t}-\frac{1}{4} u_{x x x}+3 u u_{x}-\frac{3}{4} \partial_{x}^{-1} u_{y y}=0,
$$

соответствует зависимости нормировки задачи (1) от дополнительных параметров, выраженной уравнениями

$$
D_{x} Q=0, \quad D_{y} Q=0, \quad D_{t} Q=0,
$$

здесь $\chi_{1}=D_{x} \psi, u=\chi_{11_{x}} / \psi_{1}, r=\psi_{1_{x}}$. 
ПримеР 2. Введем более сложную зависимость $Q_{p}$ от параметров $x, y, t$ :

$$
L_{1} Q \equiv D_{x} Q=0, \quad L_{2} Q \equiv\left(D_{t}-D_{y}\right) Q=0
$$

Переопределенная система линейных уравнений, получаемая из $\bar{\partial}$-проблемы, имеет следующий вид:

$$
\begin{gathered}
\left(D_{y} D_{x}-D_{x}^{3}-W_{1} D_{x}^{2}-W_{2} D_{x}\right) \psi=0 \\
W_{1}=-2 \frac{\psi_{1 x}}{\psi_{1}}, \quad W_{2}=\frac{\psi_{1}}{\psi_{1}}-3 \frac{\psi_{1 x x}}{\psi_{1}}-2\left(\frac{\psi_{2}}{\psi_{1}}\right)_{x}+4\left(\frac{\psi_{1 x}}{\psi_{1}}\right)^{2} ; \\
\left(D_{t}-D_{y}-D_{x}^{3}-W_{3} D_{x}^{2}-W_{4} D_{x}\right) \psi=0 \\
W_{3}=-1-3 \frac{\psi_{1}}{\psi_{1}}, \quad W_{4}=-3\left(\frac{\psi_{2}}{\psi_{1}}\right)_{x}-3 \frac{\psi_{1 x x}}{\psi_{1}}-2 W_{3} \frac{\psi_{1 x}}{\psi_{1}} .
\end{gathered}
$$

Соответствуюшая нелинейная система состоит из уравнения КП (18) для функции

$$
u=\partial_{x} \psi_{1}+\frac{\psi_{2}}{\psi_{1}}
$$

и линейного уравнения для функции $\phi=\psi_{1}$ с коэффициентами, зависяшими от $u$,

$$
\left(A_{-}+L_{-}\right) \phi=0
$$

Здесь использованы операторы $A_{-}, L_{-}$, определенные формулами (15).

ПримеР 3. Пусть нормировка удовлетворяет системе уравнений

$$
L_{1} Q=D_{x} Q=0, \quad L_{2} Q=\left(D_{t}-D_{y}^{2}\right) Q=0
$$

Тогда получим систему нелинейных уравнений, состоящую из уравнения КП (18) и уравнения $\left(A_{-}-L_{-}^{2}\right) \phi=0$, где использованы операторы $L_{-}, A_{-}$вида $(15)$,

$$
\phi=\psi_{1}, \quad u=\partial_{x} \psi_{1}+\frac{\psi_{2}}{\psi_{1}}
$$

ПримеР 4. Возьмем два скалярных уравнения (1) с нормировками $q_{1}, q_{2}$ и решениями $\psi, \varphi$, соответственно:

$$
\left.\psi\right|_{\lambda \rightarrow \infty} \rightarrow q_{1}+\sum_{k} \frac{\psi_{k}}{\lambda^{k}},\left.\quad \varphi\right|_{\lambda \rightarrow \infty} \rightarrow q_{2}+\sum_{k} \frac{\varphi_{k}}{\lambda^{k}}
$$

Зависимость нормировок от параметров $x, y, t$ введем формулами

$$
D_{x} Q_{i}=0, \quad D_{y} Q_{i}=0, \quad D_{t} Q_{1}+Q_{2}=0, \quad i=1,2 .
$$


В этом случае вспомогательная линейная система уравнений имеет вид

$$
\begin{aligned}
&\left(D_{y}-D_{x}^{2}+2 \xi_{x} D_{x}\right) \psi=0, \quad \xi=\ln \psi_{1}, \\
&\left(D_{y}-D_{x}^{2}+2 \eta_{x} D_{x}\right) \varphi=0, \quad \eta=\ln \varphi_{1}, \\
& \varphi_{1} D_{x} \psi=\psi_{1} D_{x} \varphi \\
&\left(D_{t}-D_{x}^{3}+3 \xi_{x} D_{x}^{2}+3\left(\xi_{x x}-\xi_{x}^{2}+v_{x}\right) D_{x}\right) \psi+\varphi=0, \quad v=\frac{\psi_{2}}{\psi_{1}} .
\end{aligned}
$$

Ей соответствует нелинейная система

$$
\begin{gathered}
r_{t}-\frac{1}{4} r_{x x x}-\frac{3}{4} \partial_{x}^{-1} r_{y y}+\frac{3}{2} r_{x} \partial_{x}^{-1} r_{y}+\frac{3}{2} r^{2} r_{x}+q_{x}=0, \\
q_{y}+q_{x x}+2 q_{x} r=0, \quad r=\xi_{x}, \quad q=\frac{\phi_{1}}{\psi_{1}},
\end{gathered}
$$

решения которой связаны с решениями уравнения КП преобразованием Миуры (16).

Выписанная система имеет очевидную интерпретацию. Подставим функцию $u(16)$ в уравнение КП (18). Получим после алгебраических преобразований, что $S(\mathrm{mKP})=0$, где $S=\partial_{y}+\partial_{x}^{2}+2 r_{x} \partial_{x}$ и буквами $\mathrm{mKP}$ обозначена левая часть модифицированного уравнения КП (17). После обрашения оператора $S$ имеем следуюшую систему уравнений:

$$
\mathrm{mKP}+s=0, \quad S(s)=0,
$$

где $s$ - ядро оператора $S$. Если теперь положить $s \equiv q_{x}$, то получим из $(25)$ в точности систему (24).

Нетрудно проверить, что уравнения (24) являются условием совместности следующей переопределенной линейной системы уравнений, получаемой из системы $(23)$ :

$$
\begin{gathered}
L_{M} \Psi=0, \quad A_{1} \Psi=\left(\partial_{x} A_{M}-q \partial_{x}\right) \Psi=0, \\
\Psi=\psi \exp \left(\lambda x+\lambda^{2} y+\lambda^{3} t\right),
\end{gathered}
$$

где операторы $L_{M}, A_{M}$ имеют вид

$$
\begin{gathered}
L_{M}=\partial_{y}-\partial_{x}^{2}+2 r \partial_{x}, \quad A_{M}=\partial_{t}-\partial_{x}^{3}+3 r \partial_{x}^{2}+\frac{3}{2} v_{0} \partial_{x} \\
v_{0}=r_{x}-r^{2}+\partial_{x}^{-1} r_{y}
\end{gathered}
$$

а условие их коммутативности порождает модифицированное уравнение КП. Выпишем упомянутое условие совместности

$$
A_{1} L_{M}-L_{M} A_{1}=2 r_{x} A_{1}
$$


ПримеР 5. Введем зависимость нормировок от параметров $x, y, t$ по формулам

$$
D_{x} Q_{i}=0, \quad D_{t} Q_{i}=0, \quad D_{y} Q_{1}+Q_{2}=0, \quad i=1,2 .
$$

Решения также обозначим буквами $\psi$ и $\varphi$. Этой задаче соответствуют линейная система уравнений

$$
\begin{gathered}
\left(D_{y}-D_{x}^{2}+2 \xi_{x} D_{x}\right) \psi+\varphi=0, \quad \xi=\ln \psi_{1}, \\
\varphi_{1} D_{x} \psi=\psi_{1} D_{x} \varphi, \\
\left(D_{t}-D_{x}^{3}+3 \xi_{x} D_{x}^{2}+3\left(\xi_{x x}-\xi_{x}^{2}+v_{x}\right) D_{x}\right) \psi=0, \quad v=\frac{\psi_{2}}{\psi_{1}}, \\
\left(D_{t}-D_{x}^{3}\right) \varphi+\left(3 w D_{x}^{2}+3\left(w_{x}+\frac{\varphi_{2 x}}{\psi_{1}}-2 w \xi_{x}-w v\right) D_{x}\right) \psi=0, \quad w=\frac{\varphi_{1 x}}{\psi_{1}}
\end{gathered}
$$

и нелинейная система

$$
\begin{gathered}
r_{t}-\frac{1}{4} r_{x x x}-\frac{3}{4}\left(\partial_{x}^{-1} r_{y y}-q_{x x}+q_{y}-2 r_{x} \partial_{x}^{-1} r_{y}-2 r^{2} r_{x}-2(q r)_{x}\right)=0 \\
q_{t}-q_{x x x}+\frac{3}{2}\left(q q_{x}-q_{x} r_{x}+q_{x} \partial_{x}^{-1} r_{y}-q_{x} r^{2}-2 q_{x x} r\right)=0 \\
r=\xi_{x}, \quad q=\frac{\varphi_{1}}{\psi_{1}}
\end{gathered}
$$

причем решения последней связаны с решениями уравнения КП (18) соотношением

$$
2 u-q=\partial_{y}^{-1} r+r_{x}+r^{2}
$$

Выписанная система является условием совместности переопределенной линейной системы уравнений

$$
L_{1} \Psi=\left(\partial_{x} L_{M}+q \partial_{x}\right) \Psi=0, \quad A_{M} \Psi=0
$$

и имеет вид

$$
A_{M} L_{1}-L_{1} A_{M}=B L_{1}, \quad B=-3\left(r_{x} \partial_{x}+v_{0 x}\right),
$$

операторы $L_{M}, A_{M}$ определены формулами (27).

Благодарности. Автор выражает благодарность С. В. Манакову за обсуждение результатов работы. Работа была поддержана РФФИ (грант № 98-01-0525).

\section{Список литературы}

[1] В. Е. Захаров, А. Б. Шабат. Функц. анализ и его прилож. 1974. Т. 8. № 3. С. 43.

[2] В. Е. Захаров, С. В. Манаков. Функц. анализ и его прилож. 1985. Т. 19. № 2. С. 11.

[3] L. V. Bogdanov, S. V. Manakov. J. Phys. A. 1988. V. 21. P. L537.

[4] А. И. Зенчук, С. В. Манаков. ТМФ. 1995. Т. 105. № 3. С. 371.

Поступила в редакцию 21.IX.1998 г. 